\section{Perspectiva de género y vulnerabilidad en salud: ¿cuándo empezamos a incluir a los hombres?}

\section{Gender perspective and vulnerability in health: when do we start to involve men?}

\section{Sr. Editor:}

El mito que ronda ante un "naufragio" de cualquier tipo es que mujeres y niños deben ser asistidos y rescatados en primer lugar; la población restante, los hombres, adquieren el rol de valientes rescatistas que asistirán a los más desvalidos antes de pensar en su propia salvación. El imaginario de este mito devela la imposibilidad de pensar la vulnerabilidad como parte del ser masculino, o bien la obligación de ser resistente, incluso a pesar de miedos o temores que llegue a experimentar. Es decir, un hombre puede ser vulnerable en situaciones puntuales, por ejemplo si está viviendo una situación económica compleja. Sin embargo, la vulnerabilidad entendida como fragilidad o debilidad no es una característica propia del género masculino, de acuerdo a los modelos de socialización a que están expuestos muchos varones.

Según los estudios de género, cada persona que forma parte de un grupo social recibe una serie de códigos respecto a su propio rol ${ }^{1,2}$. $\mathrm{Di}$ chos códigos se convierten en un referente que guía las creencias de los individuos respecto de lo que es considerado femenino y masculino. Tradicionalmente el género masculino impone a los hombres un rol de trabajador y proveedor del hogar: un hombre debe trabajar y sostener económicamente a su familia, procurando que su mujer y sus hijos no tengan necesidades materiales. Por ende, la masculinidad tradicionalmente es concebida en la esfera de lo público, un hombre que sale del hogar para cumplir con el rol de proveeduría material.

A nivel mundial se observa una diferencia entre los promedios de esperanza de vida de hombres y mujeres ${ }^{3}$ las mujeres viven aproximadamente 6 a 7 años más que los hombres, situación que se repite en Latinoamérica y en Chile. Algunos estudios han permitido suponer que dichas diferencias se deberían a diferencias en los roles de género otorgados culturalmente, más que a diferencias biológicas o sexuales $^{4,5}$. En un esfuerzo por entender la salud masculina en Chile, el Ministerio de Salud realizó en el año 2011 un perfil de salud de los hombres chilenos utilizando los datos epidemiológicos disponibles ${ }^{6}$. El documento recoge información sobre mortalidad, morbilidad y factores de riesgo en salud. Sin embargo, pese a que este reporte nos permite conocer las principales problemáticas en salud de los hombres chilenos, no presenta un enfoque de género que permita analizar los resultados. Por ende, los datos descritos resultan insuficientes para entender las necesidades, percepciones, motivaciones y comportamientos de este grupo, incluyendo los componentes culturales involucrados en sus creencias en salud.

El sistema de salud chileno actualmente no presenta una política o estrategia dirigida a la salud de los hombres. De hecho, la forma de organizar sus intervenciones en salud ha distanciado a la población masculina ${ }^{4}$, quienes usualmente acuden al sistema de salud formal en caso de enfermedad ya avanzada o en casos de accidentes que ameriten una visita improvisada a las salas de urgencia. La desconexión del sistema de salud chileno con el grupo de hombres, sumado a mandatos de género que les imponen a ellos "mostrarse fuertes" y resistir el dolor, sitúan a este grupo en una posición de vulnerabilidad en salud.

Muchos hombres chilenos se encuentran interesados en su propia salud ${ }^{4}$, pero en muchas ocasiones desconocen la oferta que el sistema de salud tiene para ellos, reconociendo que muchas veces no se sienten "invitados" a ser parte de las intervenciones propuestas y desconociendo los programas a los cuales podrían acceder, lo cual impacta en el acceso de este grupo al sistema de salud formal.

Ante la pregunta ¿por qué en Chile los hombres son vulnerables en relación a su propia salud?, la respuesta necesariamente tiene relación con los aprendizajes de género, con la falta de visibilidad por parte del macro sistema, las políticas públicas y los sistemas de salud formales, por la ausencia de programas pensados desde una lógica de género, por perpetuar fantasías respecto a los privilegios sociales del ser hombre y, por ende, por no indagar en sus necesidades, por pensarlos fuertes y no dar espacio para que muestren su debilidad y fragilidad. Ahora bien, ¿esto convierte a los hombres en víctimas de su socialización de género? Probablemente no en muchos casos, sin embargo, es difícil que un grupo que ha sido cuestionado por 
los privilegios sociales de dominación, perciba la necesidad de cuestionar su propia fragilidad. La trampa ideológica de representar el "sexo fuerte" o el "macho" se convierte en una amenaza que se perpetúa como privilegio, afectando no solo a los hombres, sino a familias y comunidades en su totalidad.

Una de las posibles vías para avanzar en el diseño de estrategias que apunten a mejorar el bienestar en salud de los hombres en Chile, involucra principalmente a los trabajadores de la salud. Son ellos quienes debieran sumar a sus esfuerzos la comprensión de las creencias específicas de la población respecto al proceso de salud y enfermedad, comprendiendo las necesidades de la población y adaptando sus estrategias de prevención y promoción desde un conocimiento real del otro. En este contexto, es importante continuar indagando en los fenómenos asociados a la salud masculina como parte central de los estudios asociados a género y salud, visibilizando algunas de la problemáticas que afectan a los hombres en el contexto del rol de género que les ha sido asignado, y cómo este rol pudiera convertirse en un impedimento para la adopción de conductas de autocuidado.

Margarita Bernales, PhD

Salud Comunitaria, Universidad de Auckland, Nueva Zelanda. Docente Investigadora, Facultad de Medicina Universidad del Desarrollo, Santiago de Chile.

\section{Referencias}

1. Courtenay W. Constructions of masculinity and their influence on men's well-being: a theory of gender and health. Social Science \& Medicine [Internet]. 2000; 50: [1385-401 pp.]. Available from: http://www.postpartummen.com/pdfs/SS\&M.PDF.

2. Connell R. The Social Organization of Masculinity. Masculinities. Cambridge: Polity Press; 1995. p. 67-86.

3. World Health Organization [WHO]. World Health Statistics 2014 2014. Available from: http://www.who. int/gho/publications/world_health_statistics/2014/en/.

4. Bernales M. Chilean men's health: perceptions and relationships with the public health care system. Auckland: The University of Auckland; 2013.

5. Cameron E, Bernardes J. Gender and disadvantage in health: men's health for a change. Sociology of Health \& Illness 1998; 20 (5): 673-93.

6. Chilean Ministry of Health. Situación Actual de la Salud del Hombre en Chile [Current situation of men's health in Chile] 2011. Available from: http://www.minsal.gob. cl/portal/url/item/b7e8f68be82d7f2fe040010165013351. pdf.

Financiamiento: Esta carta forma parte del proyecto Fondecyt N 11130042 "Desarrollando Inteligencia en Salud Pública para inmigrantes en Chile: un estudio multi-métodos" proveniente de la Comisión Nacional de Investigación Científica y Tecnológica del Gobierno de Chile.

Correspondencia a:

Dra. Margarita Bernales

margaritabernales@udd.cl 